University of Nebraska - Lincoln

DigitalCommons@University of Nebraska - Lincoln

$5-1-1998$

\title{
THE SPIN POLARIZED BAND STRUCTURE OF STRAINED THIN FILMS OF GADOLINIUM
}

\author{
C. Waldfried \\ University of Nebraska-Lincoln \\ Elio Vescovo \\ Brookhaven National Laboratory, vescovo@bnl.gov \\ Peter A. Dowben \\ University of Nebraska-Lincoln, pdowben@unl.edu
}

Follow this and additional works at: https://digitalcommons.unl.edu/physicsdowben

Part of the Physics Commons

Waldfried, C.; Vescovo, Elio; and Dowben, Peter A., "THE SPIN POLARIZED BAND STRUCTURE OF STRAINED THIN FILMS OF GADOLINIUM" (1998). Peter Dowben Publications. 163.

https://digitalcommons.unl.edu/physicsdowben/163

This Article is brought to you for free and open access by the Research Papers in Physics and Astronomy at DigitalCommons@University of Nebraska - Lincoln. It has been accepted for inclusion in Peter Dowben Publications by an authorized administrator of DigitalCommons@University of Nebraska - Lincoln. 


\title{
THE SPIN POLARIZED BAND STRUCTURE OF STRAINED THIN FILMS OF GADOLINIUM
}

\author{
C. WALDFRIED*, E. VESCOVO**, P. A. DOWBEN*
}

*Department of Physics \& Astronomy and the Center for Materials Research and Analysis, University of Nebraska-Lincoln, Lincoln, NE 68588-0111.

**National Synchrotron Light Source, Brookhaven National Laboratory, Upton, N.Y. 11973.

\section{ABSTRACT}

The magnetic properties of strained thin films of gadolinium are characterized by a wave vector and thickness dependence of the exchange splitting. The spin-resolved band structure has been mapped by spin polarized photoemission, and provides considerable insight into the relationship between magnetism of local moment systems, and band structure.

\section{INTRODUCTION}

For more than 30 years theorists [1] have predicted that the magnetic coupling and exchange splitting of elemental local moment magnetic systems is wave vector dependent and strongly affected by the band structure. We have now the first direct experimental evidence of wave vector dependent exchange splitting in an elemental local moment system. The spin-polarized band structure of strained thin films of gadolinium is found to exhibit a compelling wave vector and thickness dependence of the magnetic exchange splitting [2].

Repeatedly there has been a tendency to assume that both, magnetic coupling and the correlation energy $U$, are wave vector independent. The possibility that the magnetic coupling or the correlation energy is anything other than a scalar is often ignored. Some recent studies [3] even suggest a wave vector independent exchange splitting of gadolinium. There is no a priori reason for this [1].

Magnetic anisotropy along distinct crystallographic directions is a consequence of spin-spin interactions (or so called dipole-dipole interactions) and spin-orbit interactions. The spin-orbit interactions make the spin sensitive to the crystal lattice and are generally the dominant effect [4,5]. Gadolinium is a ferromagnet where coupling to the crystal lattice is traditionally expected through spin-spin coupling (crystal field effects dominate) while spin-orbit coupling is expected to be weak because of the half filled $4 \mathrm{f}$ shell. The strong dipole coupling will also manifest in an anisotropy relative to specific crystal directions (magneto-crystalline anisotropy), and in 
conjunction with some spin-orbit contributions can result in a wave vector dependence of the magnetic coupling. This also implies that the magnetic coupling of an elemental local moment system is expected to not only depend upon lattice spacing and bond angles but, as we will discuss in this paper, may also be strongly affected by the valence electron localization and the spin-polarized band structure. It is important to understand the significance of wave vector dependent magnetic behavior.

\section{EXPERIMENT}

Key to our understanding of the relationship between electronic structure and rare earth magnetism may be in the investigation of surfaces other than the basal plane (0001) [6] and in strained lattices. Since the electronic structure is expected to be different for different surfaces, so should be the magnetic behavior. Strained thin films of gadolinium with an increased lattice constant of approximately $4 \%$ as compared to $\mathrm{Gd}(0001)$ have been obtained by growing $\mathrm{Gd}$ on the corrugated surface of $\mathrm{Mo}(112)$ [2]. Ultra-thin ( $3 \mathrm{ML}<\mathrm{d}<10 \mathrm{ML}$ ) and thin (d >10 ML) films
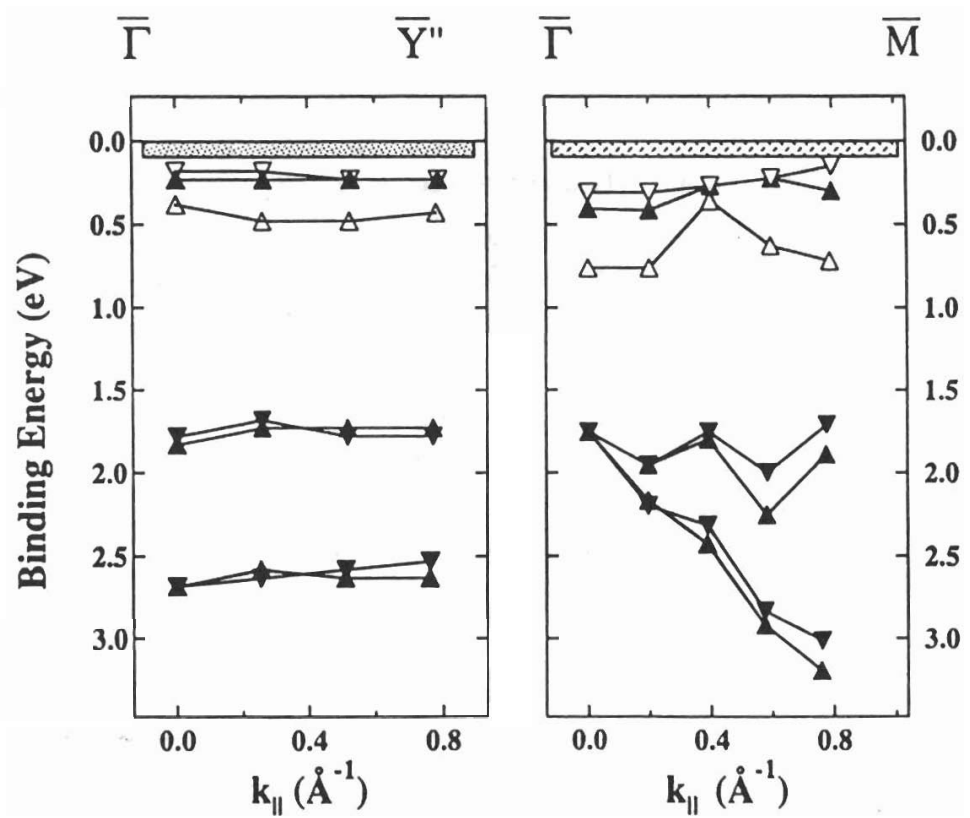

Fig. 1: Spin resolved band structure of strained Gd(1012) (left) and Gd(0001) (right) at approximately $145 \mathrm{~K}$. The filled symbols indicate bands with bulk character while the open symbols mark the binding energy positions of states with surface character. The spin resolved band structure indicates majority $(\Delta)$ and minority $(\nabla)$ bands. The hatched region near the Fermi level indicates the limited resolution. 
of Gd order in well defined rectangular and hexagonal surface unit cells, that resemble strained $\mathrm{Gd}(1012)$ and strained $\mathrm{Gd}(0001)$, respectively. Spin-polarized photoemission experiments were carried out at the new U5UA undulator beamline of the National Synchrotron Light Source (NSLS) at the Brookhaven National Laboratory in Upton, NY. The details of the experimental setups are described elsewhere [7,8]. The surface and bulk character of the bands has been determined from chemisorption studies and photon energy dependence, while the symmetry of the bands has been ascertained from the light polarization dependence.

\section{RESULTS}

The electronic band structure represents a key factor for the description of the magnetic behavior. Based on indirect measurements it has been postulated [9-12] that the exchange splitting of a local moment magnetic system is not the same for all points in the Brillouin zone, but has a strong wave vector dependence. This has been suggested by theory for Gd [13] and is clearly the case for thin films of strained $\mathrm{Gd}(0001)$ (Fig. 1b). It can be seen that the Gd $5 \mathrm{~d}_{\mathrm{xz}, \mathrm{yz}}$ or $5 \mathrm{~d}_{\mathrm{x}}{ }^{2}-\mathrm{y}^{2}$ bulk bands at $1.8 \mathrm{eV}$ below $E_{F}$ at $\bar{\Gamma}$ disperse and split along $\overline{\Gamma \mathrm{M}}$ of the hexagonal surface Brillouin zone, while at the same time the exchange splitting gradually increases.

The wave vector dependent magnetic behavior of the Gd bulk bands becomes even more clear when plotting two indicators of magnetism - exchange splitting and spin asymmetry - for

Fig. 2: The exchange splitting (top) and spin asymmetry minus background (bottom) of the bulk spin subbands as a function of wave vector. Data is shown for a $4 \mathrm{ML}$ thick film (open symbols) and a $40 \mathrm{ML}$ thick film (filled symbols) of strained Gd. The data points have been extracted from spin-polarized photoemission spectra.

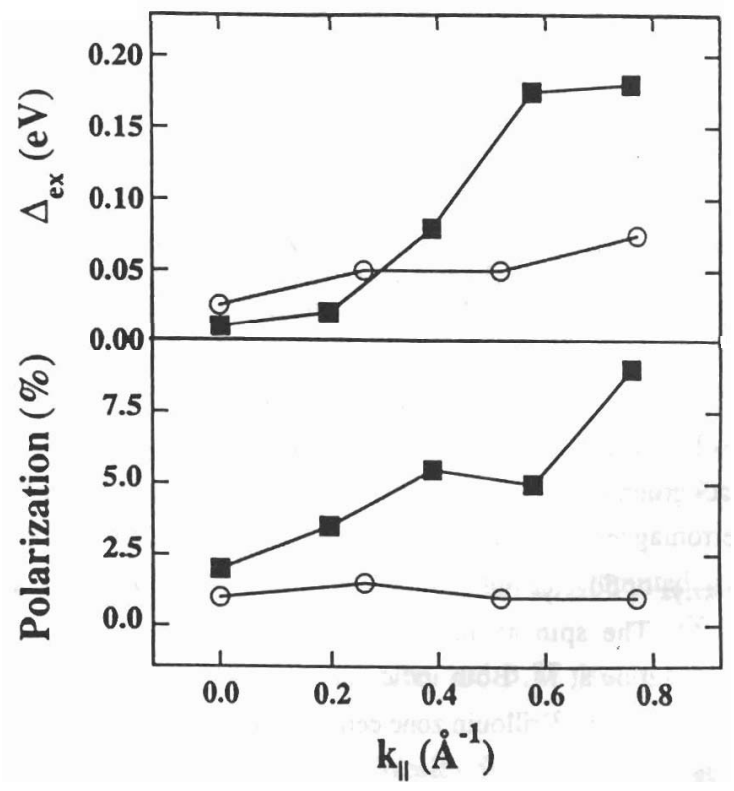




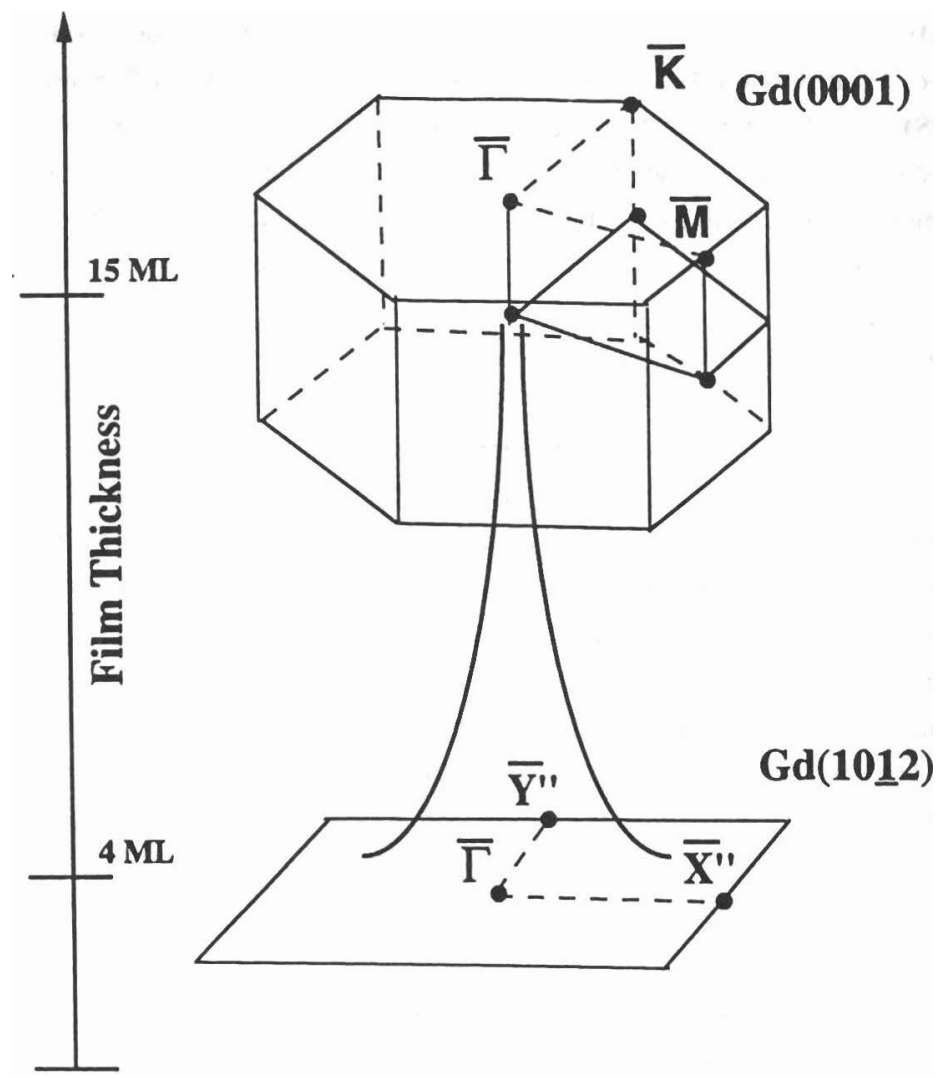

Fig. 3: Schematic of the Brillouin zone volume in which the bulk bands are paramagnetic, as function of film thickness. The Brillouin zone changes from a rectangular shape that resembles strained Gd(1012) (bottom) to the hexagonal structure of strained Gd(0001) (top).

different points of the surface Brillouin zone (Fig. 2). While the magnitude of the measured exchange splitting is an indirect indication of the Gd 5d moment, the polarization (above background) provides insight into the extent of the magnetic activity (Stoner-like and rigid band ferromagnetism). The $\Delta_{\mathrm{ex}}$ vs. $\mathrm{k}$ plot (Fig. 2) confirms the increase in exchange splitting of the Gd $5 d_{x z, y z}$ or $5 d_{x^{2}-y^{2}}$ bulk bands with increasing wave vector from less than $0.05 \mathrm{eV}$ at $\bar{\Gamma}$ to $0.2 \mathrm{eV}$ at $\bar{M}$. The spin asymmetry behaves similarly, with minimal asymmetry at $\bar{\Gamma}$ and large polarization at $\bar{M}$. Both indicators suggest a change of the bulk bands from paramagnetic-like behavior at the Brillouin zone center $\bar{\Gamma}$ to strong ferromagnetism at the zone edge $\bar{M}$. 
The spin-polarized band structure and the wave vector dependent exchange splitting are dominated by increasing electron localization for the thinner strained gadolinium films. In Fig. Ia we plot the spin-polarized band structure of an ultra-thin strained Gd film ( $d \approx 4 \mathrm{ML}$ ) from $\bar{\Gamma}$ to $\bar{Y}^{\prime}$ ' of the corresponding rectangular surface Brillouin zone (see Fig. 3). The bands show diminished dispersion. The exchange splitting of the bulk bands is negligible nearly throughout the Brillouin zone, with some indication for exchange splitting in the vicinity of the Brillouin zone edge (Fig. 2). The minimal exchange splitting, accompanied by a spin polarization that is very close to that of the background (Fig. 2), also indicates paramagnetic bulk band behavior over nearly the entire Brillouin zone.

\section{CONCLUSIONS}

Our thickness dependent spin-polarized band structure of strained thin films of gadolinium indicates that with decreasing film thickness the bulk bands become more and more localized and occupy increasingly more paramagnetic volume in the bulk Brillouin zone. In other words, the bulk bands take an increasingly "passive" role in the thinner films, while in contrast the surface remains magnetically "active" This is indicated by the significant exchange splitting (Fig. 1a) and large spin asymmetries of the surface in the ultra-thin films. The wave vector and thickness dependent distribution of para- and ferromagnetic bulk band behavior in the Brillouin zone of strained Gd is schematically shown in Fig. 3.

\section{ACKNOWLEDGMENTS}

This work was supported by NSF through grant \# DMR-92-21655 and DMR-94-96131. The experiments were carried out at the National Synchrotron Light Source which is funded by the DOE. The authors like to thank Dulip Welipitiya, and Tara McAvoy for their assistance in the experiments undertaken as part of this work.

\section{REFERENCES:}

1. R. E. Watson, and A. J. Freeman, Phys. Rev. Lett. 14, 695 (1965); R. E. Watson, and A. J. Freeman, Phys. Rev. 152, 566 (1966); R. E. Watson, A. J. Freeman, and S. Koide, Phys. Rev. 186, 625 (1969); R. E. Watson, and A. J. Freeman, Phys. Rev. 178, 725 (1969); R. E. Watson, and A. J. Freeman, J. Appl. Phys. 39, 1100 (1968).

2. C. Waldfried, D. Welipitiya, T. McAvoy, E. Vescovo, and P. A. Dowben, submitted to Phys. Rev. Lett.

3. Bongsoo Kim, A. B. Andrews, J. L. Erskine, Kwang Joo Kim, and B. N. Harmon, Phys. Rev. Lett. 68 (1992) 1931.

4. B. D. Cullity, Introduction to Magnetic Materials (Addison-Wesley Series in Metallurgy and Materials, Reading, Mass., 1972). 
5. S. V. Vonsovskii, Magnetism (John Wiley and Sons, NewYork, 1974).

6. S. D. Barrett, Surf. Sci. Reports 14 (1992) 271

7. E. Vescovo, et. al., Activity Report 1996, Nat. Synch. Light Source, A-25 (1997).

8. P. D. Johnson et. al., Rev. Sci. Instrum. 63, 1902 (1992); J. Unguris, D. T. Pierce, and R. J. Calotta, Rev. Sci. Instrum. 57, 1314 (1986).

9. Dongqi Li, Jiandi Zhang, P. A. Dowben, and M. Onellion, Phys. Rev. B 45 (1992) 7272 .

10. Dongqi Li, Jiandi Zhang, P. A. Dowben, Rong-Tzong Wu, and M. Onellion, J. Phys. Condens. Matter 4 (1992) 3929.

11. M. Donath, Appl. Phys. A 49, 351 (1989).

12. V. Korenman, R. E. Prange, Phys. Rev. Lett. 44, 1291 (1980); V. Korenman, Metallic Magnetism, ed. by H. Capellmann (Springer, Berlin 1987)

13. W. Nolting, T. Dambeck and G. Borstel, Z. Phys. B94 (1994) 409; W. Nolting, G. Borstel, T. Dambeck, T. Fauster and A. Vega, J. Magn. Magn. Mat. 140-144 (1995) 55. 